\title{
Wii Balanced Board-Based Utilization for the Human Body Center of Pressure for the Falling Detection via Multivariate Empirical Mode Decomposition and Multivariate Multi-scale Entropy
}

\author{
Jiann-Shing Shieh ${ }^{1}$, Ting-Hui Li1 ${ }^{1}$, Chih-Dao Chen ${ }^{2}$, Maysam F. Abbod ${ }^{3}$ \\ 1 Department of Mechanical Engineering and Innovation Center for Big Data and Digital Convergence, Yuan \\ Ze University, Taoyuan, Chung-Li 32003, Taiwan. \\ 2 Department of Family Medicine, Far Eastern Memorial Hospital, No.21, Sec. 2, Nanya S. Rd., Banciao Dist., \\ New Taipei City 220, Taiwan. \\ ${ }^{3}$ Department of Electronic and Computer Engineering, Brunel University London, Uxbridge UB8 3PH, UK. \\ * Corresponding author. Email: jsshieh@saturn.yzu.edu.tw \\ Manuscript submitted October 12, 2017; accepted January 23, 2018. \\ doi: 10.17706/ijbbb.2018.8.3.164-171
}

\begin{abstract}
Center of pressure (COP) evaluation is one of important parameters in the fall detection algorithm for the elder people. The Wii balance board (WBB) (Nintendo Co., Ltd., Redmond, WA 98052), originally an accessory of a game controller for the Wii video game console, is a potential device that can be utilized for the human balance evaluation system. Initially, this study evaluates the WBB signal in comparison to the AMTI (Advanced Mechanical Technology Inc., Newton, MA, USA) as the reference. Initially, the experiment is conducted for three different conditions; open eye, close eye and one-foot stand for five volunteers. The mean of correlation coefficient between WBB and AMTI is over 0.98 on X-direction and over 0.99 on Y-direction. The multivariate multi-scale entropy (MMSE) and multivariate empirical mode decomposition (MEMD) are used for the signal feature evaluations. The results show that complexity indexes (CI) from the MSE-based algorithms have significant results only between young and elderly groups and young and elderly-fall groups. However, there are no significant differences on between elderly and elderly-fall groups. In conclusion, this study shows that the WBB can be applied as the balance-measuring device. Meanwhile, further investigations need to be conducted for the advanced algorithms to fall detection for the elderly people.
\end{abstract}

Key words: Center of pressure, Wii balance board, multivariate multi-scale entropy, multivariate empirical mode decomposition, complexity index.

\section{Introduction}

Falling is one of the crucial concerns for the elderly. Figuring out the chance of fall from the elderly could be essentially important for the fall detection condition. In further, the human balance evaluation by investigating the center of pressure (COP) is fundamental in fall detector [1], [2]. The body movement will affect the anterior-posterior (A-P) and medial-lateral (M-L). Several studies have been conducted for the balance analysis. A study about the relationship of the center of gravity of body position and the COP has been conducted by Benda et al. [3]. Recently, Lu et al. utilized the accelerometer sensor for the COP evaluation [4]. 
For the balance evaluation, the balance measurement system should be well investigated. The most of the balance-measuring devices are economically expensive and physically heavy. The Wii balance board (WBB) is originally an accessory of a game controller for the Wii video game console. It was developed by on July 2007 by Nintendo (Nintendo Co., Ltd., Redmond, WA 98052). Several studies have evaluated the utilization of WBB. McGough et al. investigated the real-time weight bearing asymmetry [5]. Gil-Gómez et al. utilized the WBB for the acquired brain injury [6]. Meanwhile, Young, W et al. used the WBB for the standing balance [7]. In advanced, Huurnink et al. conducted a WBB-based study for the postural investigation with the COP evaluation [8].

Our study has two major parts; the device and the balance-related algorithms. The WBB is utilized as the balance-measuring machine with comparison to the AMTI (Advanced Mechanical Technology Inc., Newton, MA, USA) as the reference. The multivariate multi-scale entropy (MMSE) and multivariate empirical mode decomposition (MEMD) are used for the signal feature evaluations.

\section{Device, Experimental Design and Methods}

The dimension of WBB is relatively portable; $511 \mathrm{~mm} \times 316 \mathrm{~mm} \times 53.2 \mathrm{~mm}$ respectively for the width, length and depth. WBB is powered by four AA-size batteries and has four 16-bit pressure sensors, marked by S1, S2, S3 and S4 as shown on Fig.1. The program interface developed by Japanese Rehabilitation Tool Study Group (http://www.eonet.ne.jp/ rpt/) is used for the data collection via Bluetooth connection. This program is able to save A/P-direction and M/L-direction signals, and draw COP. For the actual location, the ratio should multiply the size of balance board. The length between the sensors are $42 \mathrm{~cm}$ and $24 \mathrm{~cm}$. Meanwhile, the center of WBB is zero that left to right is -21 to $21 \mathrm{~cm}$ and top to bottom is -12 to $12 \mathrm{~cm}$. The evaluation of the COP is calculated as follows;

$$
\begin{gathered}
K x=\frac{S 1+S 2}{S 3+S 4} \\
K y=\frac{S 1+S 4}{S 2+S 2} \\
C O P x=\frac{K x-1}{K x+1} *(-21) \\
\text { COPy }=\frac{K y-1}{K y+1} *(-12)
\end{gathered}
$$

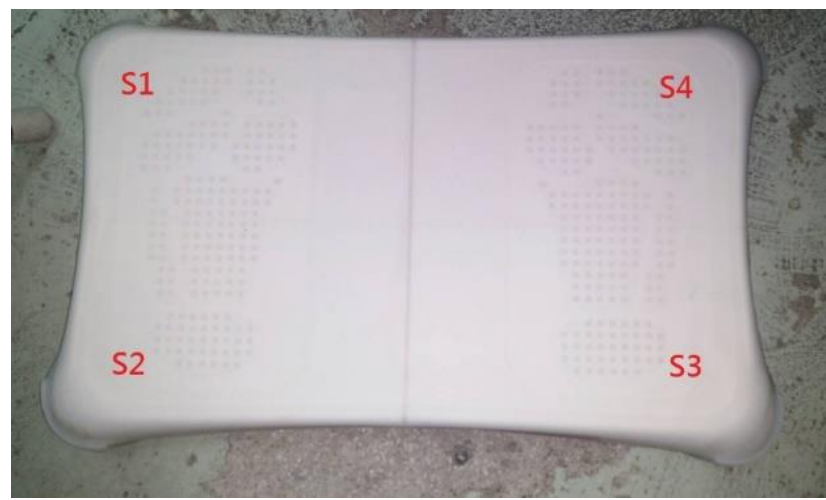

Fig. 1. Wii balanced board sensors. 
For the experimental result comparison of the WBB, the AMTI is utilized for the reference as shown on Fig. 2. AMTI can evaluate three moments and three forces for the X-Y-Z axes by the following equations;

$$
\begin{gathered}
C O P x=\frac{M y+F x * Z_{0}}{F z}+X_{0} \\
C O P y=\frac{M x+F y * Z_{0}}{F z}+Y_{0}
\end{gathered}
$$

where the $F_{x}, F_{y}$ and $F_{z}$ are the forces from each axis, respectively. Furthermore, the $M x, M y$ and $M z$ are the moments from each axis, respectively. The $X_{o}$ and $Y_{o}$ are the initial positions from the subject and $Z_{o}$ is the top of the plate.

Five volunteers are involved to conduct three different conditions; open eye, close eye and one-foot stand. Each condition will be repeated for five times for every volunteer. The correlation coefficient for X- and Y-axis are evaluated.

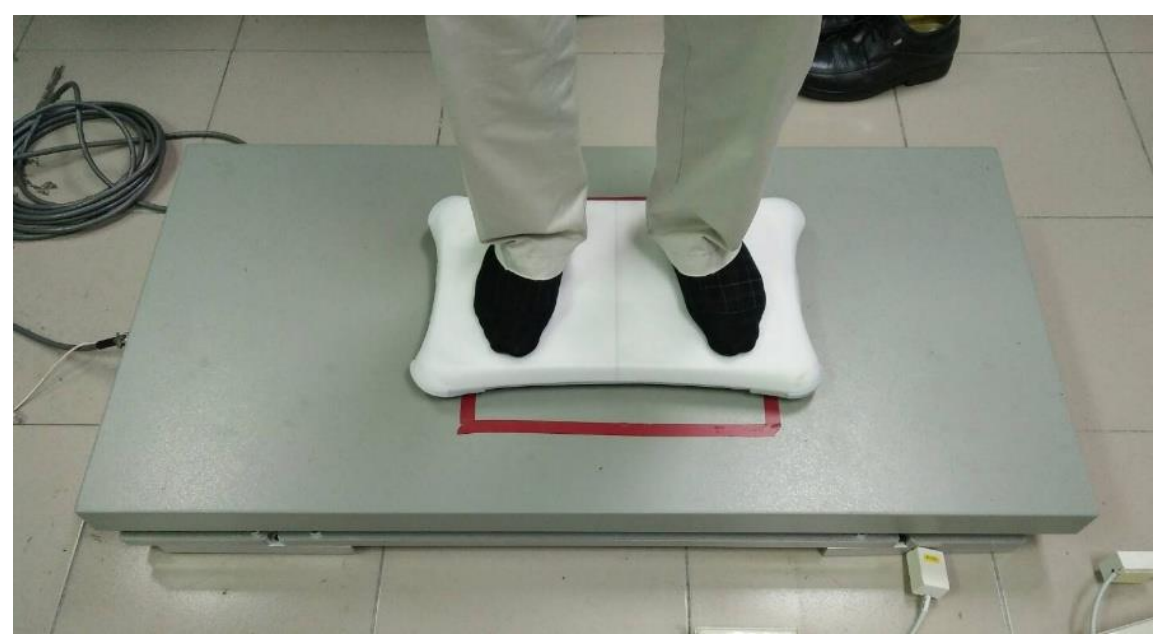

Fig. 2. Comparing WBB and AMTI.

The first applied algorithm is the multivariate multi-scale entropy (MMSE). Initially Richman et al. proposed the sample entropy (SE) for analyzing the complex system [9] and the modified SE, namely MSE, multi scale entropy [10], the MMSE is proposed [11]. The time series $\left\{\mathrm{y}^{(\tau)}\right\}$ is reconstructed with the scale factor, $\tau$. For the evaluation of the COP complexity, the complexity index (CI) is evaluated by calculating the area under MSE curve.

For the filtering algorithm, the empirical mode decomposition (EMD) is utilized. EMD is applied for the non-linear and non-stationary signal [12]. It works by finding the local minima and maxima in order to generate the lower and upper envelopes, respectively. It also will decompose the original time series data to several intrinsic mode functions (IMFs). The EMD equation is shown as following;

$$
X(t)=\sum_{i=1}^{n} c_{i}+r_{n}
$$

where $X(t)$ is the original signal, $c_{i}$ is the $i$-IMF and $r_{n}$ is the residual.

However, the original EMD algorithm has the mode mixing problem. The ensemble EMD (EEMD) was developed by Wu et al. to overcome the mode mixing problem by adding the noise to the original signal [13]. In advanced, the multivariate EMD (MEMD) is working by not only adding the noise, but also evaluating the 
multivariate input signal [14] and has the advantages to the multi-channel signal [15]. The modified MMSE, MEMD-enhanced MMSE, is further evaluated. This study originally proposed by [16]. The MEMD-enhanced MMSE works by replacing the course graining-based time series to $C_{n}$ which is equation (12) or equation (13).

$$
\begin{gathered}
C_{n}=\sum_{j=n}^{J} c_{j} \\
C_{n}=\sum_{j=1}^{J-n+1} c_{j}
\end{gathered}
$$

where the MEMD decomposes the original time series into the number of IMFs, $c_{j}$ denotes $j$-th IMF and $n$ is $[1, \mathrm{j}]$.

\section{Result and Discussion}

Via the comparison result between WBB and AMTI signal, it can be seen in Fig. 3. The correlation coefficient for both WBB and AMTI is evaluated for different conditions as shown in Tables 1-3. The experiment is conducted for three different conditions; open eye, close eye and one-foot stand for five volunteers. The mean of correlation coefficient between WBB and AMTI is over 0.98 on X-direction and over 0.99 on Y-direction. In order to compare the MMSE and the MEMD-enhanced MMSE algorithms, the IMF 4 to IMF 7 are selected to the constructed signal. For the visualization, the comparisons are shown in Figs. 4-6. For the statistical analysis, the t-test evaluation is utilized to evaluate the $\mathrm{CI}$ as shown in Table 4 for 14 young, 47 elderly, and 23 elderly-fall volunteers. The results show that complexity indexes from the MSE-based algorithms have significant results $(\mathrm{P}<0.05)$ only between young and elderly groups, and young and elderly-fall groups. However, there is no significant difference between elderly and elderly-fall groups.
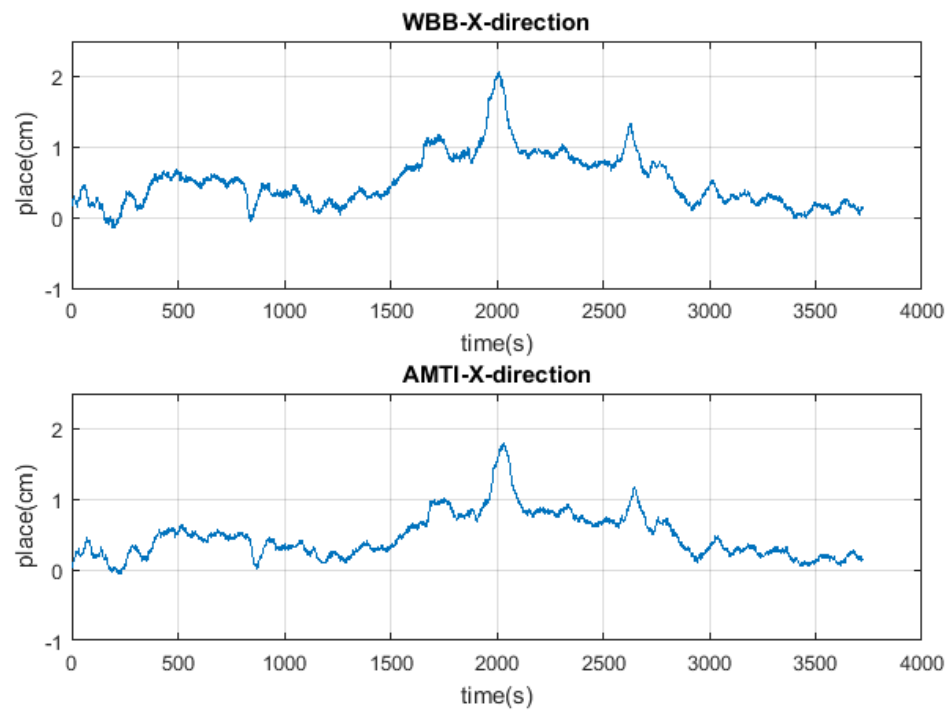

Fig. 3. Resampled X-direction signals comparison between WBB and AMTI.

Table 1. Correlation Coefficient between WBB and AMTI - Open Eye

\begin{tabular}{|c|c|c|}
\hline Volunteer & $X$ & $Y$ \\
\hline A & $0.981 \pm 0.011$ & $0.995 \pm 0.002$ \\
\hline
\end{tabular}




\begin{tabular}{|c|c|c|}
\hline B & $0.987 \pm 0.009$ & $0.990 \pm 0.006$ \\
\hline C & $0.969 \pm 0.013$ & $0.991 \pm 0.006$ \\
\hline D & $0.978 \pm 0.007$ & $0.992 \pm 0.006$ \\
\hline E & $0.965 \pm 0.027$ & $0.987 \pm 0.010$ \\
\hline Average mean \pm SD & $0.978 \pm 0.008$ & $0.992 \pm 0.004$ \\
\hline
\end{tabular}

Table 2. Correlation Coefficient between WBB and AMTI - Close Eye

\begin{tabular}{|c|l|l|}
\hline Volunteer & \multicolumn{1}{|c|}{ X } & \multicolumn{1}{c|}{$Y$} \\
\hline A & $0.995 \pm 0.001$ & $0.995 \pm 0.004$ \\
\hline B & $0.971 \pm 0.028$ & $0.992 \pm 0.013$ \\
\hline C & $0.977 \pm 0.011$ & $0.995 \pm 0.003$ \\
\hline D & $0.979 \pm 0.010$ & $0.991 \pm 0.009$ \\
\hline E & $0.947 \pm 0.023$ & $0.985 \pm 0.004$ \\
\hline Average mean \pm SD & $0.976 \pm 0.018$ & $0.990 \pm 0.007$ \\
\hline
\end{tabular}

Table 3. Correlation Coefficient between WBB and AMTI - One Foot Standing

\begin{tabular}{|c|l|l|}
\hline Volunteer & \multicolumn{1}{|c|}{ X } & \multicolumn{1}{c|}{$Y$} \\
\hline A & $0.990 \pm 0.007$ & $0.993 \pm 0.004$ \\
\hline B & $0.987 \pm 0.007$ & $0.996 \pm 0.001$ \\
\hline C & $0.985 \pm 0.012$ & $0.996 \pm 0.002$ \\
\hline D & $0.985 \pm 0.020$ & $0.994 \pm 0.003$ \\
\hline E & $0.937 \pm 0.116$ & $0.992 \pm 0.009$ \\
\hline Average mean \pm SD & $0.980 \pm 0.022$ & $0.994 \pm 0.005$ \\
\hline
\end{tabular}

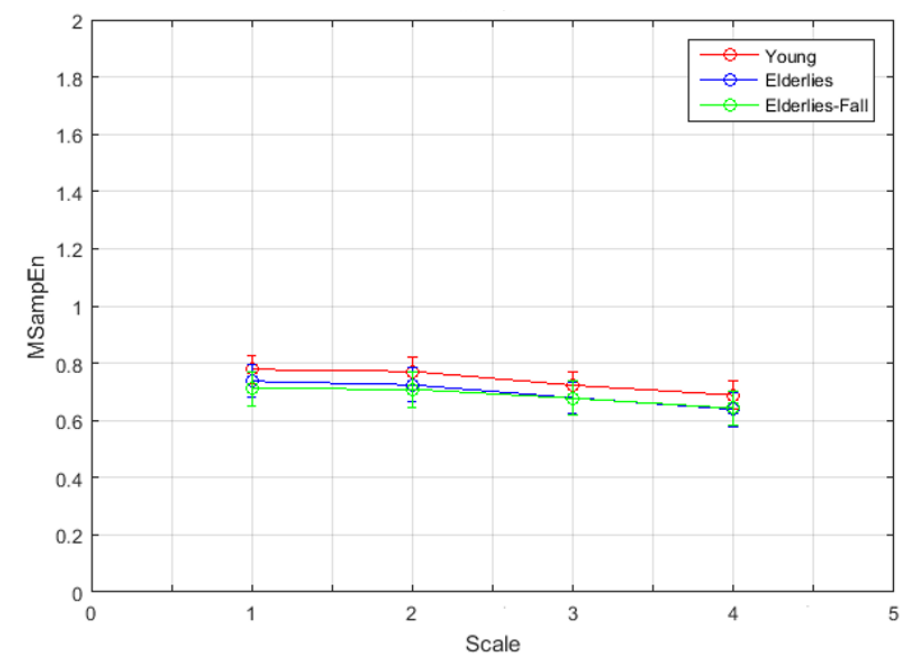

Fig. 4. MMSE IMF 4 to 7 with $\mathrm{m}=2, \mathrm{r}=0.15$. 


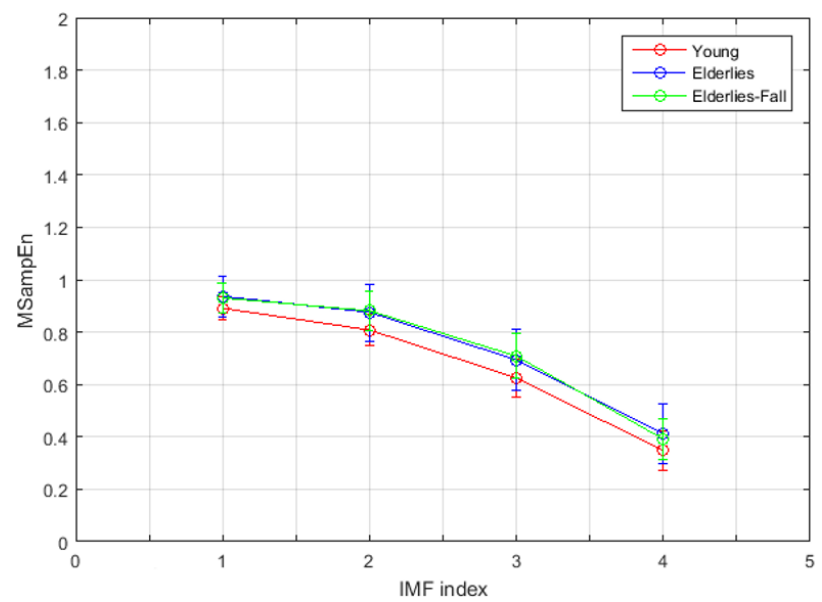

Fig. 5. MEMD-enhance MMSE uses equation (8) and IMF 4 to 7 with $m=2, r=0.15$.

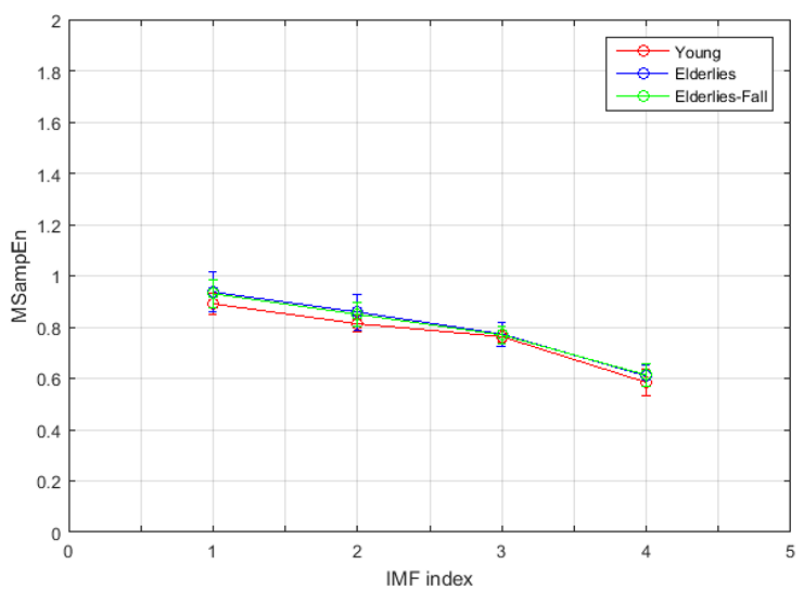

Fig. 6. MEMD-enhance MMSE uses equation (9) and IMF 4 to 7 with $m=2, r=0.15$.

Table 4. Statistical Results between Each Group for 14 Young, 47 Elderly, and 23 Elderly-Fall Volunteers

\begin{tabular}{cccc}
\hline Methods & $\begin{array}{c}\text { Young vs. } \\
\text { Elderly }\end{array}$ & $\begin{array}{c}\text { Young vs. } \\
\text { Elderly-Fall }\end{array}$ & $\begin{array}{c}\text { Elderly vs. } \\
\text { Elderly-Fall }\end{array}$ \\
\hline MMSE & $5.77 \mathrm{E}-05$ & $1.41 \mathrm{E}-05$ & 0.169 \\
$\begin{array}{c}\text { MEMD-enhanced MMSE equation } \\
\text { (8) }\end{array}$ & $4.78 \mathrm{E}-04$ & $5.60 \mathrm{E}-06$ & 0.443 \\
$\begin{array}{c}\text { MEMD-enhanced MMSE equation } \\
(9)\end{array}$ & $5.59 \mathrm{E}-04$ & $1.61 \mathrm{E}-05$ & 0.308 \\
\hline
\end{tabular}

\section{Conclusion}

In conclusion, this study shows that the WBB can be applied as the balance-measuring device. Meanwhile, the results show that complexity indexes from the MMSE and MEMD-enhanced MMSE algorithms have significant results only between young and elderly groups, and young and elderly-fall groups. However, there is no significant difference between elderly and elderly-fall groups. Further investigations need to be conducted for the advanced algorithms to fall detection for the elderly people. Also, the number of volunteers should be increased to prove which algorithms are more suitable to fall detection.

\section{Acknowledgment}

This research was supported by the two centers of Innovation Center for Biomedical and Healthcare Technology, and Innovation Center for Big Data and Digital Convergence, Yuan Ze University, Taiwan. 


\section{References}

[1] Pajala, S., Era, P., Koskenvuo, M., Kaprio, J., Törmäkangas, T., \& Rantanen, T. (2008). Force platform balance measures as predictors of indoor and outdoor falls in community-dwelling women aged 63-76 years. The Journals of Gerontology Series A: Biological Sciences and Medical Sciences, 63(2), 171-178.

[2] Costa, M., Priplata, A. A., Lipsitz, L. A., Wu, Z., Huang, N. E., Goldberger, A. L., \& Peng, C. K. (2007). Noise and poise: enhancement of postural complexity in the elderly with a stochastic-resonance-based therapy. EPL (Europhysics Letters), 77(6), 68008.

[3] Benda, B. J., Riley, P. O., \& Krebs, D. E. (1994). Biomechanical relationship between center of gravity and center of pressure during standing. IEEE Transactions on Rehabilitation Engineering, 2(1), 3-10.

[4] Lu, S. H., Shieh, J. S., \& Hansen, C. (2016). Applied a multi-scale entropy algorithm to analyze dynamic COP signal via accelerometer sensor. Ubiquitous Intelligence \& Computing, Advanced and Trusted Computing, Scalable Computing and Communications, Cloud and Big Data Computing, Internet of People, and Smart World Congress (UIC/ATC/ScalCom/CBDCom/IoP/SmartWorld), 2016 Intl IEEE Conferences (pp. 127-132). IEEE.

[5] McGough, R., Paterson, K., Bradshaw, E., Bryant, A., \& Clark, R. (2011). The use of Nintendo Wii balance boards for real-time investigation of weight bearing asymmetry. ISBS-Conference Proceedings Archive (Vol. 1, No. 1).

[6] Gil-Gómez, J. A., Lloréns, R., Alcañiz, M., \& Colomer, C. (2011). Effectiveness of a Wii balance board-based system (eBaViR) for balance rehabilitation: A pilot randomized clinical trial in patients with acquired brain injury. Journal of Neuroengineering and Rehabilitation, 8(1), 30.

[7] Young, W., Ferguson, S., Brault, S., Craig, C. (2011). Assessing and training standing balance in older adults: a novel approach using the 'Nintendo Wii'Balance Board. Gait \& Posture, 33(2), 303-305.

[8] Huurnink, A., Fransz, D. P., Kingma, I., \& Dieën, J. H. (2013). Comparison of a laboratory grade force platform with a Nintendo Wii Balance Board on measurement of postural control in single-leg stance balance tasks. Journal of biomechanics, 46(7), 1392-1395.

[9] Richman, J. S., \& Moorman, J. R. (2000). Physiological time-series analysis using approximate entropy and sample entropy. Am J Physiol Heart CircPhysiol, 278, H2039-H2049.

[10] Costa, M., Goldberger, A. L., \& Peng, C. K. (2002). Multiscale entropy analysis of complex physiologic time series. Physical review letters, 89(6), 068102.

[11] Ahmed, M. U., \& Mandic, D. P. (2011). Multivariate multiscale entropy: A tool for complexity analysis of multichannel data. Physical Review E, 84(6), 061918.

[12] Huang, N. E., Shen, Z., Long, S. R., Wu, M. C., Shih, H. H., Zheng, Q., Yen, N. C., Tung, C. C., \& Liu, H. H. (1998). The empirical mode decomposition and the Hilbert spectrum for nonlinear and non-stationary time series analysis. Proceedings of the Royal Society of London A: Mathematical, Physical and Engineering Sciences, 454(1971), 903-995.

[13] Wu, Z., \& Huang, N. E. (2009). Ensemble empirical mode decomposition: a noise-assisted data analysis method. Advances in Adaptive Data Analysis, 1(01), 1-41.

[14] Rehman, N., \& Mandic, D. P. (2010). Multivariate empirical mode decomposition. Proceedings of the Royal Society of London A: Mathematical, Physical and Engineering Sciences, 466(2117), 1291-1302.

[15] Rehman, N., \& Mandic, D. P. (2011). Filter bank property of multivariate empirical mode decomposition. IEEE Transactions on Signal Processing, 59(5), 2421-2426.

[16] Ahmed, M. U., Rehman, N., Looney, D., Rutkowski, T. M., \& Mandic, D. P. (2012). Dynamical complexity of human responses: A multivariate data-adaptive framework. Bulletin of the Polish Academy of Sciences: Technical Sciences, 60(3), 433-445. 


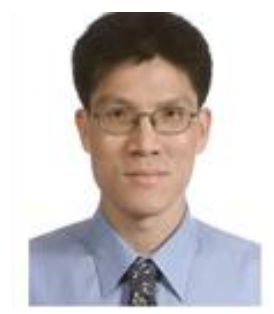

Jiann-Shing Shieh received the B.Sc. and M.Sc. degrees in chemical engineering from the National Cheng Kung University, Taiwan, ROC, in 1983 and 1986, respectively, and the Ph.D. degree in automatic control and systems engineering from the University of Sheffield, U.K., in 1995.

He was a postdoctoral research associate in the Department of Automatic Control and Systems Engineering at the University of Sheffield, from 1995 to 1996, working on a hierarchical fuzzy logic-based support system project. Also, he was a postdoctoral research associate in the Center for Biomedical Engineering, College of Medicine, National Taiwan University, from 1996 to 1998, working on an anesthesia systems engineering and its clinical applications. Currently, he is professor of Mechanical Engineering at the Yuan Ze University. His current research interests include intelligent analysis and control, bio-signal processing, critical care medicine monitoring and control, pain model and control, medical automation, dynamic cerebral autoregulation research, and brain death index research.

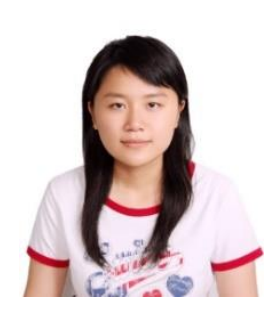

Ting-Hui Lee received the master degree in mechanical from the Yuan Ze University, Taiwan, ROC, in 2017. Her master thesis was working on complexity of human balance and the method of signal analyzing.

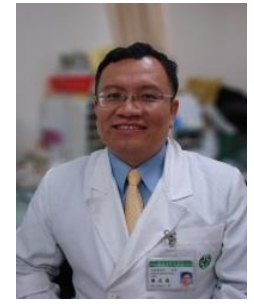

Chih-Dao Chen received the M.D. degree from the Kaohsiang Medical University, Taiwan, ROC, in 1995, respectively, and the Ph.D. degree in preventive medicine from the Institute of Epidemiology and Preventive Medicine, National Taiwan University, in 2008. He received family medicine resident training in Kaohsiung Medical University Hospital, from 1995 to 1998. He was a visiting staff of family medicine in Kaohsiung Medical University Hospital, from 1998 to 1999. Now, he is the Chief of Family Medicine Department in Far Eastern Memorial Hospital in New Taipei City, Taiwan, ROC.

He focused on the study of colorectal cancer natural history. He was a Postdoctoral Research Associate in Institute of Epidemiology and Preventive Medicine, National Taiwan University, from 2008, working on a Epidemiology and Prevention for Metabolic Syndrome and Colorectal Cancer. Currently, he is a Associate Professor of Health Care Management in Oriental Institute of Technology. His current research interests include family medicine, preventive medicine, cancer screening, geriatric medicine, adolescent health, and hospice care.

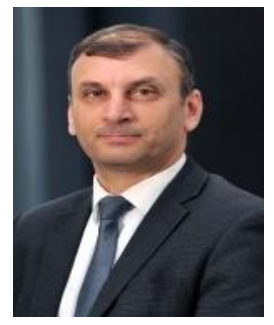

Maysam Abbod (PI) is a reader in intelligent system in the Department of Electronic and Computer Engineering at Brunel. He has published 117 refereed journal papers, 16 chapters in edited books and 122 refereed conference papers. He is a CI on EU funded project, CI on Innovate UK project, and British Council Exchange projects. His main research interests are in intelligent systems for modelling, control and optimisation. Developed intelligent systems were applied to industrial and biomedical modelling, and computer control of manufacturing systems. The main research areas are: Intelligent systems for text mining and classification, hybrid modelling techniques (multivariate/time series) and data mining and data driven modelling techniques. 